\title{
Eficacia y seguridad del dietiletilbestrol, en el tratamiento del cáncer de próstata resistente a la castración
}

\section{Effectiveness and Safety of Diethylstilbestrol in the Treatment of Castration Resistant Prostate Cancer}

\author{
Jorge Eduardo Gómez Meza ${ }^{1}$ Rodolfo Varela ${ }^{2}$ Angie Ramírez ${ }^{3}$ \\ ${ }^{1}$ Departamento de Urología, Fundación Universitaria Sanitas, Clínica \\ Universitaria Colombia, Bogotá, Colombia \\ 2 Departamento de Urología, Jefe del servicio de Urología Oncológica, \\ Instituto Nacional de Cancerología, Ciudad de México, CDMX, \\ México \\ 3 Department of Medico General, Instituto Nacional de Cancerología, \\ Bogotá, Colombia \\ Urol Colomb 2018;27:42-47.

\begin{abstract}
Address for correspondence Jorge Eduardo Gómez Meza, MD, Calle 131 a \#9-59 Apartamento 201 Torre F, Conjunto el Vergel del Country,
\end{abstract} \\ Bogotá, Colombia (e-mail: jorgemeza99@gmail.com).
}

\section{Resumen}

Palabras Claves

- cáncer de próstata

- resistencia a castración

- antígeno prostático específico

- dietiletilbestrol

- efectos adversos

- terapia hormonal
Introducción y objetivos Existen múltiples opciones de tratamiento en pacientes con cáncer de próstata resistente a la castración, entre ellas el Dietilestilbestrol (DES) sin embargo, su uso es controversial. Este estudio tiene como objetivo determinar la eficacia y seguridad del DES, en el tratamiento de pacientes con cáncer de próstata resistente a la castración en nuestra población.

Métodos y Materiales Se realizó un estudio de corte transversal, incluyendo los pacientes con cáncer de próstata resistente a la castración que recibieron tratamiento con DES. Se realizó un análisis demográfico, bivariado, tomando como desenlace la respuesta del PSA (Ausente, Completa o Parcial), el tiempo medio de progresión del PSA y la presencia de eventos adversos asociados al medicamento.

Resultados Noventa y un pacientes incluidos al final del estudio. La distribución de respuesta del PSA fue así: Respuesta en 57 (63,7\%) pacientes, (Completa 28\% - 31,1\% y parcial 29\% - 32,2\%). Ausente en 33 (36,7\%) pacientes. El análisis bivariado no evidenció asociación entre las variables y los desenlaces propuestos. El tiempo medio de progresión del PSA fue de 10,43 meses (Log-rank $p=0.001$ ), no se encontraron diferencias estadísticamente significativas para el tiempo medio a progresión en asociación con la respuesta al PSA (respuesta o ausente y la presencia de enfermedad metastásica), Log-rank $p=0,789$, Log-rank $p=0,218$, Log-rank $p=0,780$ respectivamente. La tasa de complicaciones asociadas a DES fue del $4,4 \%$ y correspondió en todos los casos a trombosis venosa profunda.

Conclusiones El DES en pacientes con cáncer de próstata resistente a la castración continúa siendo una herramienta de tratamiento eficaz y con baja tasa de eventos adversos en nuestra población. received

September 29, 2016

accepted

December 7, 2017
DOI https://doi.org/ 10.1055/s-0038-1639583. ISSN 0120-789X. eISSN 2027-0119.
Copyright (c) 2018, Sociedad Colombiana License terms de Urología. Publicado por Thieme Revinter Publicações Ltda., Rio de Janeiro, Brazil. Todos los derechos reservados. 


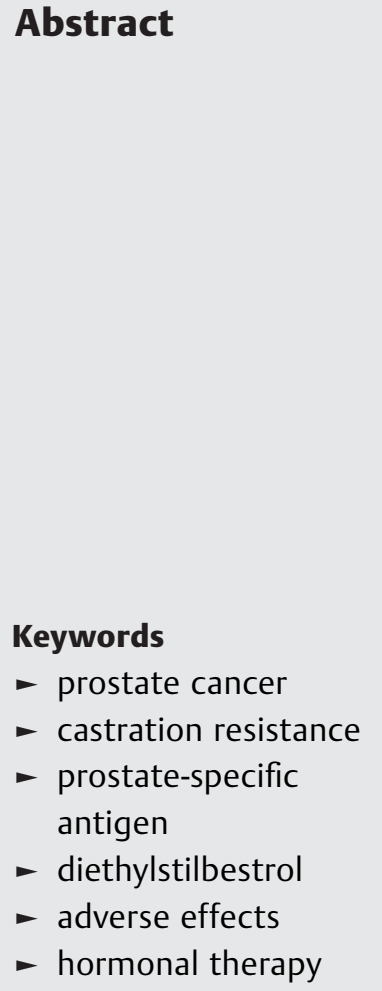

Introduction and Objectives There are multiple treatment options in patients with castration-resistant prostate cancer, including diethylstilbestrol (DES), but its use is controversial. This study aims to determine the efficacy and safety of DES in the treatment of patients with castration resistant prostate cancer in our population.

Methods and Materials A cross-sectional study was performed, including patients with castration resistant prostate cancer who were treated with DES. A demographic analysis was performed, bivariate analysis, taking as outcome PSA response (Complete or partial), the median time to PSA progression and the presence of adverse events associated with the drug.

Results 91 patients included at the end of the study. The distribution of PSA response was so; Response in 57 (63.7\%) patients (Full 28 to $31.1 \%$ and partial 29 to $32.2 \%$ ). Absent in 33 (36.7\%) patients. Bivariate analysis evidenced no association between the variables and proposed outcomes. The median time to PSA progression was 10.43 months ( $\log$-rank $p=0.001)$, no statistically significant differences in the average time to progression was found in association with PSA response (response or absent and the presence of disease metastatic), Log-rank $p=0.789$, log-rank $p=0.218$, log-rank $p=0.780$ respectively. The rate of complications associated with DES was $4.4 \%$ and corresponded in all cases to deep vein thrombosis.

Conclusions DES in patients with resistant prostate cancer castration continued to be an effective choice of tool of treatment with a low rate of adverse events in our population.

\section{Introducción}

El cáncer de próstata ( $\mathrm{CaP})$, se constituye como el segundo cáncer más común en hombres. ${ }^{1,2}$ En el escenario del paciente con cáncer de próstata con resistencia a la castración (CPRC), existen múltiples estudios con diversos medicamentos $\mathrm{y}$ estrategias de secuenciación. En la actualidad, el uso de un fármaco olvidado como el dietiletilbestrol (DES), se encuentra cada vez más limitado debido a la incidencia de complicaciones de predomino cardiovascular y al entendimiento de la biología del receptor de andrógenos que ha favorecido la aparición de nuevos medicamentos como Abiraterona, Enzalutamida y Radio 223 con un mejor perfil de seguridad, pero a expensas de un alto costo y aumento en la supervivencia que varía entre 4 y 10 meses. $^{3-10}$

El DES es una alternativa en el manejo escalonado de esos pacientes, con un costo significativamente menor y posibles resultados de supervivencia equiparables. ${ }^{6,11,12}$

Este estudio tiene como objetivo determinar la eficacia y seguridad del DES en pacientes con CPRC en un centro de referencia.

\section{Metodología}

Se realizó un estudio observacional, descriptivo, de corte transversal con muestreo no probabilístico, en pacientes con CPRC metastásico y no metastásico y tratados con DES $1 \mathrm{mg}+$ ASA 100mg entre marzo del 2013 y septiembre del 2014, en el Instituto Nacional de Cancerología de Bogotá,
Colombia. Se excluyeron los pacientes con datos incompletos en la historia clínica y con un seguimiento menor a 6 meses.

Los desenlaces principales evaluados fueron la respuesta serológica del antígeno prostático específico (PSA) y el tiempo a progresión bioquímica. Adicionalmente, se evaluó la tasa de complicaciones asociados con el tratamiento.

Para el seguimiento bioquímico se tuvieron en cuenta las siguientes variables: PSA al inicio de tratamiento; respuesta del PSA: respuesta completa (RCPSA) definido como la reducción mayor o igual al 50\% del PSA con relación al PSA inicial, respuesta parcial (RPPSA) definido como la reducción del PSA menor al 50\% en relación con el PSA inicial; tiempo a progresión (TMP) definido como el tiempo en el cual se evidencia progresión del PSA (aumento del PSA en un 50\% sobre el nadir en pacientes con RCPSA y el aumento del $25 \%$ sobre el nadir en pacientes con RPPSA). Todas las respuestas del PSA y evidencia de progresión fueron confirmadas con un segundo valor.

\section{Resultados}

Se encontraron 197 registros de pacientes con diagnóstico de CPRC que recibieron DES $1 \mathrm{mg}+\mathrm{ASA} 100 \mathrm{mg}$. De esos se excluyeron 107 pacientes que no cumplieron los criterios planteados: 19 pacientes por datos incompletos en el registro de historia clínica, 1 paciente por intolerancia al medicamento posterior a la primera dosis y 87 pacientes por seguimiento clínico menor a 6 meses, para un total de 90 pacientes incluidos para el análisis (-Fig. 1). (imagen adjunta). 


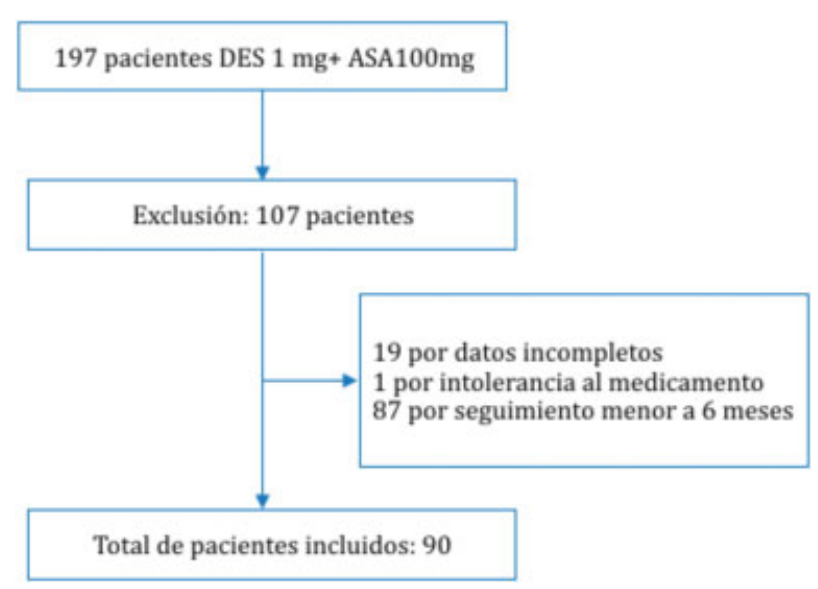

Fig. 1 Selección de pacientes.

La edad media de los pacientes fue 75,3 años (52-90 años). El estadio clínico tumoral fue cTx (42,2\%), seguido del estadio cT3a (23,3\%). En cuanto al puntaje de Gleason bióptico se encontró que un $43,3 \%$ tenían un valor desconocido y un $27,8 \%$ tuvieron Gleason $>7$. Se encontró enfermedad metastásica en el 74,4\% de los casos. La media de fosfatasa alcalina fue de $306 \mathrm{U} / \mathrm{L}$ y la media de PSA al inicio de fue $327,4 \mathrm{ng} / \mathrm{ml}$ y el $91,1 \%$ de los pacientes tenían castración con orquidectomía simple bilateral.

El 95,6\% de los pacientes recibió tratamiento con antiandrogenos siendo la bicalutamida el más frecuente. El $47,8 \%$ de los pacientes tuvieron al menos 2 tratamientos hormonales previos al DES. (-Tabla $\mathbf{1}$ )

Se evidenció progresión bioquímica en $73 \%$ de los pacientes, con un tiempo medio a la progresión de 10,43 meses. En el 52,3\% de los pacientes se realizó cambio de

Tabla 1 Características demográficas

\begin{tabular}{|l|l|l|}
\hline Característica & & $\mathbf{n}=\mathbf{9 0}(\%)$ \\
\hline Edad (años) & $75,3(52-90)$ & \\
\hline PSA(ng/mL) & $327,4(3,5-3329)$ & \\
\hline Testosterona & $6,099(0-104)$ & \\
\hline Estadio clínico & $\mathrm{T} 1$ & $6(6,5 \%)$ \\
\hline & $\mathrm{T} 2$ & $11(12,2 \%)$ \\
\hline & $\mathrm{T} 3$ & $21(23,3 \%)$ \\
\hline & $\mathrm{T} 4$ & $14(15,6 \%)$ \\
\hline & $\mathrm{Tx}$ & $38(42,2 \%)$ \\
\hline Gleason & $<7$ & $16(17,8 \%)$ \\
\hline & 7 & $10(11,1 \%)$ \\
\hline & $>7$ & $25(27,8 \%)$ \\
\hline & Desconocido & $29(43,3 \%)$ \\
\hline Metástasis & & \\
\hline & $\mathrm{M} 1$ & $67(74,4 \%)$ \\
\hline & $\mathrm{M} 0$ & $23(25,6 \%)$ \\
\hline Seguimiento (meses) & $9,33(6-18)$ & \\
\hline
\end{tabular}

Tabla 2 Resumen de tratamientos realizados

\begin{tabular}{|l|l|l|}
\hline $\begin{array}{l}\text { Características } \\
\text { tratamiento }\end{array}$ & $\mathbf{n}=\mathbf{9 0}(\%)$ \\
\hline Tratamiento inicial & $\begin{array}{l}\text { Prostatectomía } \\
\text { radical }\end{array}$ & $16(17,8 \%)$ \\
\hline & Radioterapia & $8(8,9 \%)$ \\
\hline & $\begin{array}{l}\text { Linfadenectomía } \\
\text { clasificatoria }\end{array}$ & $2(2,2 \%)$ \\
\hline & Ninguno & $64(71,1 \%)$ \\
\hline Orquidectomía & Sí & $82(91,1 \%)$ \\
\hline Antiandrogeno & No & $8(8,9 \%)$ \\
\hline Primario & Bicalutamida & $76(88,4 \%)$ \\
\hline & Flutamida & $3(3,2 \%)$ \\
\hline Antiandrogeno & Ciproterona & $7(8,1 \%)$ \\
\hline Secundario & Flutamida & $33(36,7 \%)$ \\
\hline & Bicalutamida & $6(6,7 \%)$ \\
\hline Tratamientos & No recibió & $51(56,7 \%)$ \\
\hline previos al DES & 0 & $4(4,4 \%)$ \\
\hline & 1 & $30(33,3 \%)$ \\
\hline & 2 & $43(47,8 \%)$ \\
\hline & 3 o más & $13(14,4 \%)$ \\
\hline
\end{tabular}

terapia, siendo el tratamiento adicional más frecuentemente usado fue acetato de abiraterona (31,8\% de los casos). La tasa de complicaciones fue baja $(4,4 \%)$ y todas fueron trombosis de miembros inferiores. (-Tabla 2 )

Con respecto a la tasa de respuesta bioquímica al DES se encontró respuesta en $63,7 \%$ de los pacientes, siendo completa en 31,1\% y parcial en 32,2\% (- Tabla 3).

En el análisis bivariado y multivariado no se encontró ninguna característica asociada con la tasa de respuesta bioquímica. - Tabla 4.

El tiempo medio a la progresión bioquímica fue de 10,43 meses. No se encontró tampoco asociación entre la presencia de enfermedad metastásica, la respuesta inicial del PSA (Aumento del PSA, disminución o igual), respuesta al PSA (completa o parcial) con el tiempo a recaída bioquímica (Log-

Tabla 3 Respuesta PSA

\begin{tabular}{|l|l|l|}
\hline Desenlaces oncológicos & & \\
\hline Recaída bioquímica & Si & $46(73 \%)$ \\
\hline & No & $17(27 \%)$ \\
\hline Tiempo a la recaída meses & & $10,4(3-8)$ \\
\hline Cambio de tratamiento & Abiraterona & $28(31,8 \%)$ \\
\hline & Docetaxel & $18(20,5 \%)$ \\
\hline & No & $42(47,7 \%)$ \\
\hline Complicaciones & Sí & $4(4,4 \%)$ \\
\hline & No & $86(95,6 \%)$ \\
\hline Tipo de complicación & TVP & $4(100 \%)$ \\
\hline
\end{tabular}


Tabla 4 Análisis bivariado multivariado en relación a la respuesta de PSA con el uso de DES

\begin{tabular}{|l|l|l|}
\hline & Bivariado & Multivariado \\
\hline Edad & $p=0,477$ & $p=0,293$ \\
\hline Estadio Tumoral & $p=0,795$ & $p=0,935$ \\
\hline Puntaje de Gleason & $p=0,833$ & $p=0,524$ \\
\hline F. Alcalina & $p=0,766$ & $p=0,849$ \\
\hline Testosterona & $p=0,506$ & $p=0,573$ \\
\hline Enf. Metastasica & $p=0,766$ & $p=0,438$ \\
\hline Terapia inicial & $p=0,061$ & $p=0,061$ \\
\hline Orquidectomia & $p=0,959$ & $p=0,733$ \\
\hline No de tratamientos & $p=0,814$ & $p=0,523$ \\
\hline Análogo LHRH & $p=0,882$ & $p=0,639$ \\
\hline Antiandrógeno primario & $p=0,794$ & $p=0,690$ \\
\hline Antiandrógeno secundario & $p=0,862$ & $p=0,884$ \\
\hline PSA inicial & $p=0,232$ & $p=0,350$ \\
\hline
\end{tabular}

Abreviaturas: p, Chi-cuadrado.

rank $p=0.780 ;$ Log-rank $p=0.789 ;$ Log-rank $p=0.218$ respectivamente) (-Fig. 2-4).

\section{Discusión}

Con el análisis final del EORTC 30805, el cual comparaba la Orquidectomía con Orquidectomía mas acetato de ciproterona y DES a baja dosis ( $1 \mathrm{mg} /$ día), en pacientes con enfermedad metastásica, se evaluó la eficacia clínica y la tasa de complicaciones del DES a baja dosis. La media de seguimiento en este estudio multicentrico y prospectivo fue de 4 años, encontrando ausencia de diferencia estadísticamente significativa entre los diferentes brazos en cuanto a tiempo, progresión y supervivencia global. La incidencia de muertes relacionadas con eventos

\section{TIEMPO A RECAIDA (MESES) Survival curve}

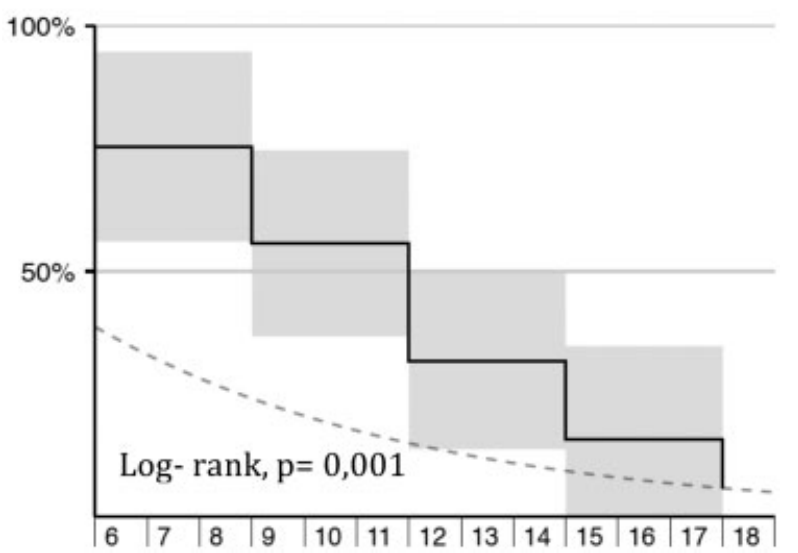

Fig. 2 Tiempo a recaída bioquímica en pacientes tratados con DES.

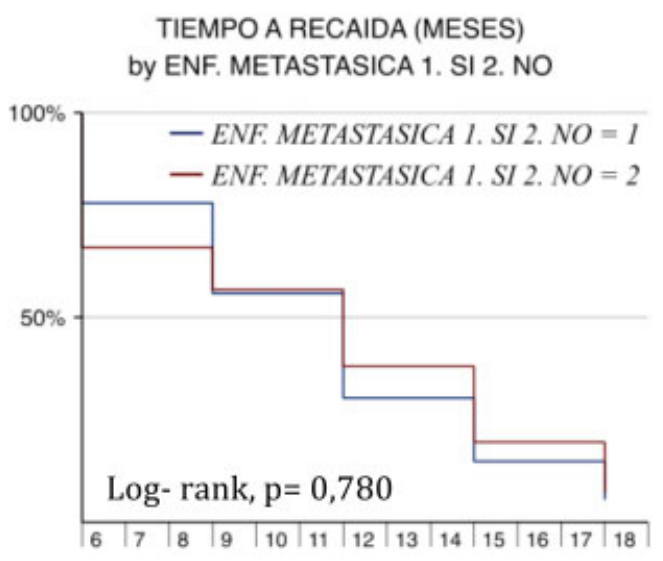

Fig.3 Estimación del tiempo de recaída con enfermedad metastásica y no metastásica.

cardiovasculares en el brazo DES fue $14,8 \%$, casi el doble que en el brazo de Orquidectomía 8,3\%. Una proporción similar fue encontrada en cuanto a la tasa de complicaciones cardiovasculares, edema y fenómenos tromboembolicos. Otro estudio, donde se incluyeron 106 pacientes con cáncer de próstata avanzado, con tiempo medio de seguimiento de 21 meses, evidenció efectos secundarios cardiovasculares en 8 de 106 pacientes. No hubo muertes relacionadas con eventos cardiovasculares y se presentó un evento trombo embolico. La supervivencia global fue comparable a otros estudios con otro manejo hormonal. En conclusión, esos estudios demuestran la eficacia clínica del DES a baja dosis, sin embargo, también evidencia la posibilidad de encontrar efectos cardiovasculares secundarios entre un $8 \%$ a un $21 \%{ }^{6,13,14}$

Nuestro estudio muestra que el uso de DES es una alternativa eficaz y segura para los pacientes con cáncer de próstata resistente a la castración, los resultados con respecto a las tasas de respuesta global que fue de un $63 \%$, respuesta total del $31,1 \%$ y parcial del $32,2 \%$ respectivamente.

La respuesta global en estudios iniciales como el de Smith y colaboradores fue de $42,8 \%$, Ramaswamy y colaboradores muestra un descenso de los niveles de PSA global del 65\% similar a nuestro estudio, con una tasa de respuesta total del $23 \%$, comparado con un $31,1 \%$ en nuestro estudio. Clemos y Wilkins muestran tasas de respuesta del $38,8 \%$ y del $28,9 \%$ respectivamente, adicionalmente discriminan las tasas de respuesta en total y parcial, mostrando para el grupo de Clemons una respuesta total en un $38 \%$ y parcial de un $32,7 \%$, el grupo de Wilkins discrimina sus tasa de respuesta en descenso del PSA entre el 25\%-50\% y $<25 \%$, los resultados fueron del $16 \%$ y $32 \%$ respectivamente.

El estudio más reciente Grenader y colaboradores es el que presenta resultados más favorables, con una tasa de respuesta total del $63 \%$ y tiempo de progresión mayor a un año en un $31 \%$ de los pacientes. Por lo tanto, nuestros resultados son consistentes con los encontrados por esos grupos. $6,13,15,16$

El tiempo medio de progresión de nuestro trabajo fue de 10,43 meses, los reportados por la literatura van de 4,6 meses 
TIEMPO A RECAIDA (MESES)

by RTA PSA 1. Completa 2. Parcial

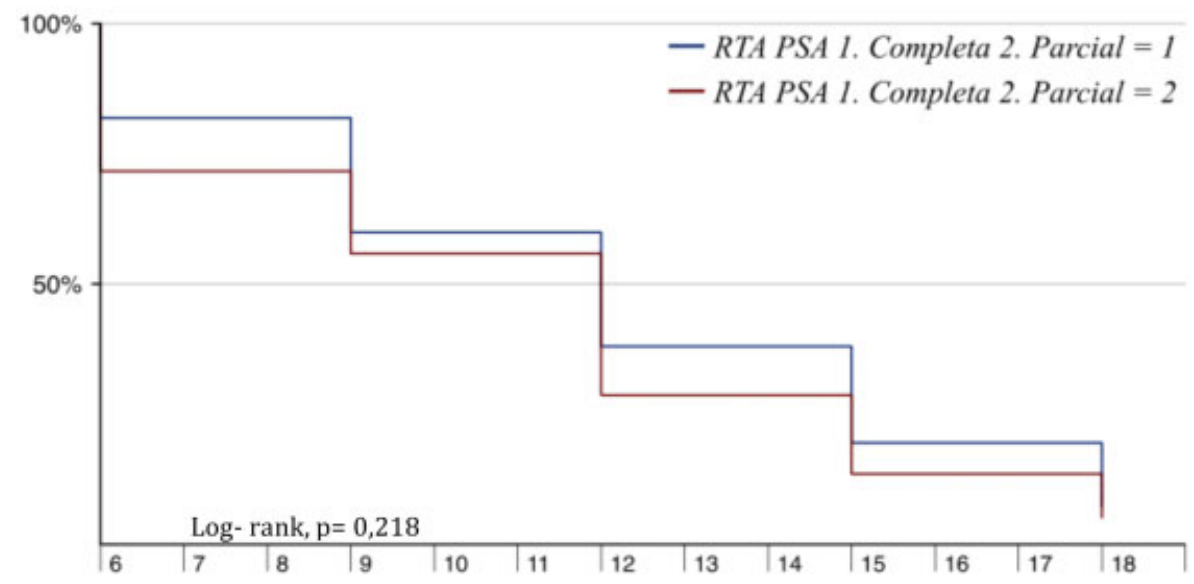

Fig. 4 Estimación del tiempo de recaída según la respuesta del PSA.

para el estudio de Wilkins y 7,5 meses para el estudio de Clemons, en ese aspecto nuestras respuestas son más favorables para los pacientes con DES. ${ }^{6}$

En el desenlace de ausencia de respuesta, nuestros resultados fueron del 35,6\%, el único estudio que presenta datos acerca de ese desenlace fue el grupo de Clemons con un $29 \%$ asociando a un aumento de los niveles del receptor estrogénico alfa el cual podría promover la progresión tumoral. ${ }^{6}$

En la evaluación de complicaciones asociadas al tratamiento con DES, el presente estudio mostró una tasa de complicación de un 4,4\% y ellas fueron tromboembólicas, la principal trombosis venosa profunda, hallazgo concordante con los estudios anteriormente mencionados. Smith y colaboradores tienen una tasa del 5\% de trombosis venosa profunda, Ramasawamy y colaboradores tienen una tasa de complicaciones del $5 \%$ y de ese porcentaje una tercera parte corresponde a complicaciones tromboembolicas, la que se presentó con dosis de $3 \mathrm{mg}$ de DES, pero con dosis de $1 \mathrm{mg}$ no hubo complicaciones reportadas. ${ }^{15,16}$

La tasa más alta reportada de complicaciones tromboembolicas fue de Grenader y colaboradores llegando a ser del 9\% relacionada directamente con la interrupción de la tromboprofilaxis. El estudio de Clemos y Wilkins respectivamente, informan tasa de complicación de un $4,8 \%$ y $3,7 \%$ que fueron tromboembolicas. ${ }^{6,14,17}$

En cuanto a los factores predictores establecidos en el estudio de Wilkins en donde encontraron la presencia de metástasis como factor predictor de repuesta al medicamento ( $p=0.045$ ), en nuestro estudio esa variable no tuvo relación con la respuesta de PSA.

Los resultados de nuestro estudio para evaluar la seguridad y eficacia de la terapia con DES en los pacientes con cáncer de próstata resistente a la castración, son comparables con los reportados previamente en la literatura, lo cual hace que implementar esa terapia sea razonable por los resultados oncológicos y menores costos, la tasa de eventos adversos es baja y puede prevenirse con la administración concomitante de aspirina.

\section{Limitaciones}

Este estudio presenta múltiples limitaciones, entre ellas la más importante, su carácter retrospectivo, el cual puede incidir en sesgos de selección y de información. Adicionalmente se observó en las historias clínicas, que muchos de los pacientes no contaban con registro de datos completos, o tenían un seguimiento y/o manejo individual que no seguía los parámetros que indica la literatura, eso fue motivo de exclusión de una gran cantidad de pacientes. Igualmente por el carácter de la recolección de los datos, no fue posible contar con seguimiento imagenológico que permitiera determinar progresión bajo este parámetro.

Por otro lado en este estudio, encontramos una baja tasa de complicaciones, la cual es similar a la descrita en la literatura por otros autores, sin embargo, ella también podría estar atribuida al subregistro en la historias clínicas.

\section{Conclusiones}

En pacientes seleccionados, el uso de DES en pacientes con cáncer de próstata resistente a la castración metastásico y no metastásico, constituye una herramienta de manejo eficaz y segura con tasa de complicaciones bajas $(4,4 \%)$ de eventos tromboembolicos como lo evidencia nuestro estudio, con una dosis de $1 \mathrm{mg}$ acompañado de ASA $100 \mathrm{mg}$ día.

Se requieren estudios prospectivos y aleatorizados, para el uso de DES en este grupo de pacientes.

\section{Referencias}

1 Saad F, Miller K. Treatment options in castration-resistant prostate cancer: current therapies and emerging docetaxelbased regimens. Urol Oncol 2014;32(02):70-79

2 Piñeros M, Gamboa O, Hernández-Suárez G, Pardo C, Bray F. Patterns and trends in cancer mortality in Colombia 1984-2008. Cancer Epidemiol 2013;37(03):233-239 
3 Ryan CJ, Smith MR, de Bono JS, et al; COU-AA-302 Investigators. Abiraterone in metastatic prostate cancer without previous chemotherapy. N Engl J Med 2013;368(02):138-148http://www. pubmedcentral.nih.gov/articlerender.fcgi?artid=3683570\&tool= pmcentrez\&rendertype $=$ abstract [Internet]

4 Carter HB, Albertsen PC, Barry MJ, et al. American Urological Association (AUA) Guideline GUIDELINE American Urological Association Early Detection of Prostate Cancer. 2013:1-28

5 Parker C, Nilsson S, Heinrich D, et al; ALSYMPCA Investigators. Alpha emitter radium-223 and survival in metastatic prostate cancer. N Engl J Med 2013;369(03):213-223

6 Clemons J, Glodé LM, Gao D, Flaig TW. Low-dose diethylstilbestrol for the treatment of advanced prostate cancer. Urol Oncol 2013; 31(02):198-204

7 Cookson MS, Roth BJ, Dahm P, et al. Castration-resistant prostate cancer: AUA Guideline. J Urol 2013;190(02):429-438

8 Aggarwal R, Weinberg V, Small EJ, Oh W, Rushakoff R, Ryan CJ. The mechanism of action of estrogen in castration-resistant prostate cancer: clues from hormone levels. Clin Genitourin Cancer 2009; 7(03):E71-E76

9 Shore ND, Cookson MS. Proper Sequencing of Treatment for Castrate Resistant Prostate Cancer. Urol Pract 2014;1(01):2-6

10 Wilkins A, Shahidi M, Parker C, et al. Diethylstilbestrol in castrationresistant prostate cancer. BJU Int 2012;110(11 Pt B):E727-E735
11 Malkowicz SB. The role of diethylstilbestrol in the treatment of prostate cancer. Urology 2001;58(02, Suppl 1):108-113

12 Scherr DS, Pitts WR Jr. The nonsteroidal effects of diethylstilbestrol: the rationale for androgen deprivation therapy without estrogen deprivation in the treatment of prostate cancer. J Urol 2003;170 (05):1703-1708

13 Oh WK. The evolving role of estrogen therapy in prostate cancer. Clin Prostate Cancer 2002;1(02):81-89

14 Turo R, Smolski M, Esler R, et al. Diethylstilboestrol for the treatment of prostate cancer: past, present and future. Scand J Urol 2014;48(01):4-14http://www.ncbi.nlm.nih.gov/pubmed/ 24256023[Internet]

15 Manikandan R, Srirangam SJ, Pearson E, Brown SCW, O’Reilly P, Collins GN. Diethylstilboestrol versus bicalutamide in hormone refractory prostate carcinoma: a prospective randomized trial. Urol Int 2005;75(03):217-221

16 Smith DC, Redman BG, Flaherty LE, Li L, Strawderman M, Pienta KJ. A phase II trial of oral diethylstilbesterol as a second-line hormonal agent in advanced prostate cancer. Urology 1998;52 (02):257-260

17 Grenader T, Plotkin Y, Gips M, Cherny N, Gabizon A. Diethylstilbestrol for the treatment of patients with castration-resistant prostate cancer: retrospective analysis of a single institution experience. Oncol Rep 2014;31(01):428-434 